\title{
Chromosome 1p31.1p31.3 Deletion in a Patient with Craniosynostosis, Central Nervous System and Renal Malformation: Case Report and Review of the Literature
}

\author{
Carlos I. Rivera-Pedroza ${ }^{a, b}$ Jimena Barraza-García a-c \\ Beatriz Paumard-Hernández ${ }^{\mathrm{a}}$ Julian Nevado ${ }^{\mathrm{a}, \mathrm{c}}$ Carlos Orbea-Gallardo ${ }^{\mathrm{d}}$ \\ Jaime Sánchez del Pozo ${ }^{\mathrm{e}}$ Karen E. Heath ${ }^{\mathrm{a}-\mathrm{c}}$ \\ a Institute of Medical and Molecular Genetics (INGEMM) and b Multidisciplinary Unit for Skeletal Dysplasias (UMDE), \\ Hospital Universitario La Paz, Universidad Autónoma de Madrid, IdiPAZ, 'Centro de Investigación Biomédica \\ en Enfermedades Raras (CIBERER), Instituto Carlos III, and d Neonatology and ${ }^{\mathrm{e}}$ Dysmorphology Units, Hospital \\ Universitario Doce de Octubre, Madrid, Spain
}

\section{Key Words}

Chromosome $1 \cdot$ Craniosynostosis · Deletion

\begin{abstract}
Interstitial deletions in the short arm of chromosome 1 are infrequent. We report a female with a 1 p31.1p31.3 deletion and cloverleaf skull, who presented with renal and central nervous system malformations, cleft palate, severe ocular anomalies, and cutis laxa, in addition to the previously described clinical data present in other cases with deletions encompassing this region, such as developmental delay, seizures, round face with a prominent nose, micro/retrognathia, half-opened mouth, short neck, hand/foot malformations, hernia, congenital heart malformations, and abnormal external genitalia. The deletion spanned $\sim 18.6 \mathrm{Mb}$ and included a total of 68 OMIM protein coding genes. We have reviewed 17 cases previously described in the literature and in DECIPHER involving the chromosomal region 1 p31.1p31.3. Only 3 of these affect the whole region, 9 are partial deletions of this region, and 5 are much smaller deletions. Taking
\end{abstract}

\section{KARGER}

(c) 2016 S. Karger AG, Basel

E-Mail karger@karger.com

www.karger.com/msy into account the MORBID ID and the haploinsufficiency score of the genes, we go on to propose which genes may explain particular clinical features observed in the patient. IL23R may be responsible for the craniosynostosis, FOXD2 for the renal anomalies, $L H X 8$ for closure defects of the palate, and ST6GALNAC3 for skin anomalies. In summary, we have identified a chromosome 1 p31.1p31.3 deletion in a patient with an atypical presentation of craniosynostosis amongst other more typical features observed in individuals with similar deletions.

(c) 2016 S. Karger AG, Basel

Genomic rearrangements refer to alterations in the genome such as duplications, deletions, insertions, inversions, and translocations. They can cause a phenotype by various molecular mechanisms, including the alteration in the copy number of genes sensitive to dosage, by dis-

\section{C.I.R.-P. and J.B.-G. contributed equally to this work.}

Karen E. Heath, PhD

Instituto de Genética Médica y Molecular (INGEMM), Hospital Universitario La Paz Paseo de la Castellana 261

ES-28046 Madrid (Spain)

E-Mail karen.heath@ salud.madrid.org 


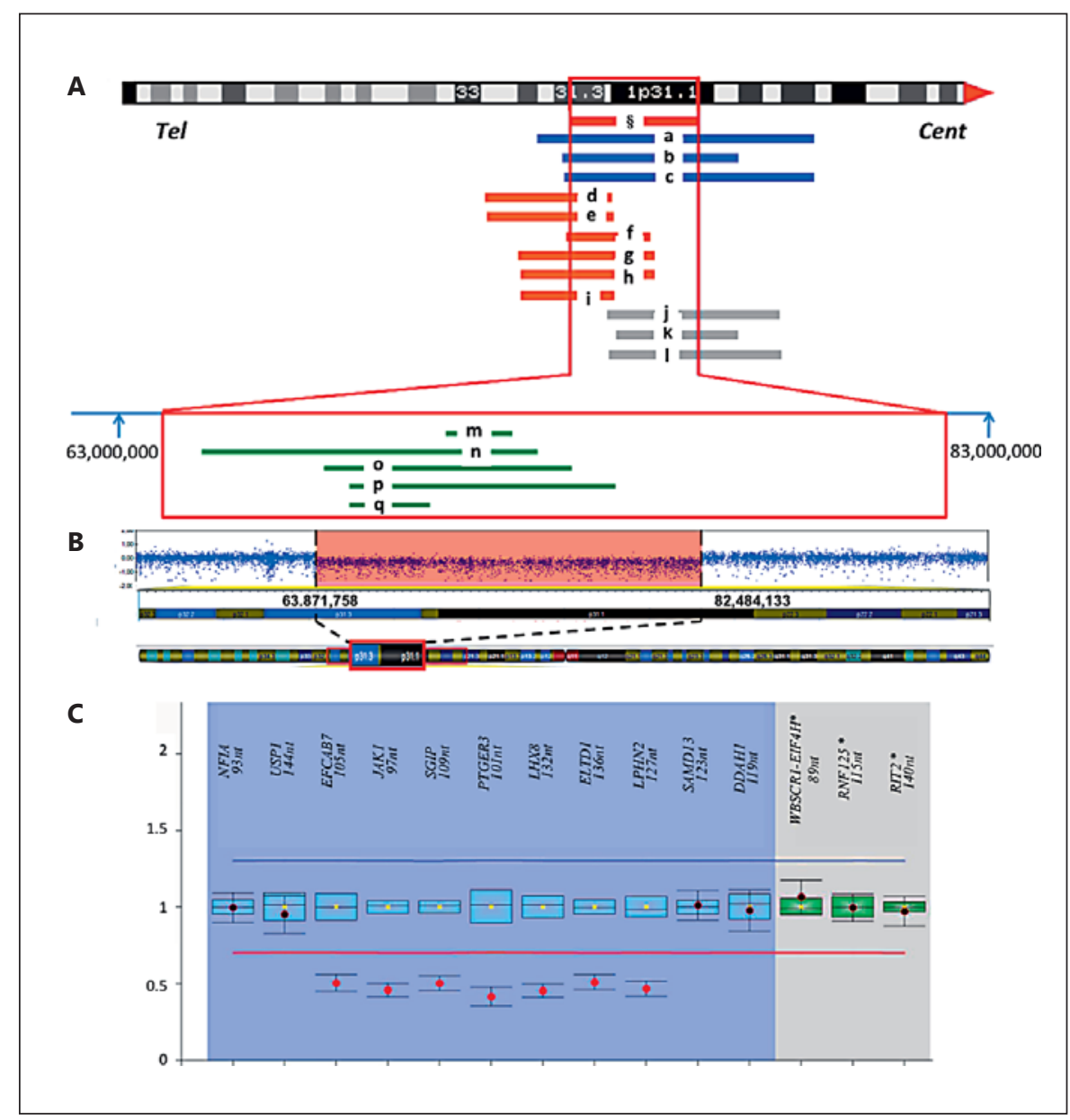

Fig. 1. Genetic analysis of the proband. A The location of the identified deletion $(\$)$ and the chromosome 1 p31 deletions reported in the literature as well as in the DECIPHER database: Bene et al., 1978 (a), Lai et al., 1991, patient 2 (b), Sivasankara et al., 1997 (c), Campbell et al., 2002, and Lu et al., 2007, patient 1 (d), Campbell et al., 2002, and Lu et al., 2007, patient 2 (e), Shaw-Smith et al., 2004 (f), Chen et al., 2011 (g), Ji et al., 2014 (h), Zinner and Batanian, 2003, and Labonne et al., 2016 (i), Petersen and Warburg, 1987 (j), Lai et al., 1991, patient 1 (k), Mircher et al., 2003 (l), Gillberg and FitzPatrick, 2010 (m), Tassano et al., 2015 (n), DECIPHER 1927 (o), DECIPHER 250300 (p), and DECIPHER 277832 (q). The colors of the deletion blocks correspond to the colors of the column heads for each patient in online supplementary table 3 . Tel $=$ Telo-

rupting a gene causing its loss of function, by unmasking a recessive allele, or by affecting a region subject to imprinting.

With the exception of the $1 \mathrm{p} 36$ syndrome (MIM 607872), interstitial deletions affecting the short arm of chromosome 1 are rare, with only 20 cases reported in the literature [Labonne et al., 2016]. Three of these are deletions encompassing the whole $1 \mathrm{p} 31.1 \mathrm{p} 31.3$ region mere; Cent $=$ centromere. B The Infinium Cyto-SNP-850K BeadChip (Illumina) showing the $\log _{2}$ ratio data. The deletion is shown between the dashed lines and corresponds to hg19 coordinates $63,871,758-82,484,133$ in 1 p31.1p31.3. C Coffalyser plot of the custom-designed chromosome 1p31.1p31.3 MLPA, confirming the presence of the deletion. Normal peaks were classified as having a ratio of $0.7-1.3$ and deletions were classified as $<0.7$. The deletion includes EFCAB7, JAK1, SGIP, PTGER3, LHX8, ELTD1, and $L P H N 2$ probes, whilst probes for NFIA and USP1 (telomeric to the deletion) as well as SMD113 and DDAH1 (centromeric to the deletion) are present in 2 copies. Asterisks represent autosomal control probes.

(fig. 1A, a-c), whilst 9 are partial deletions of this region (fig. 1A, d-1) and constitute the 1p32p31 syndrome (MIM 613735). The most frequently associated clinical findings are global developmental delay, central nervous system (CNS) malformations, dysmorphic features, and urinary tract malformations. Individuals with partial deletions present with a phenotype similar to those with a deletion of this entire region [Bene et al., 1979; Lai et al., 1991; 
Sivasankaran et al., 1997; Zinner and Batanian 2003; Shaw-Smith et al., 2004; Gillberg and FitzPatrick, 2010; Chen et al., 2011; Ji et al., 2014; Tassano et al., 2015; Labonne et al., 2016]. There are also 5 cases with significantly smaller deletions, all presenting with a milder phenotype (fig. $1 \mathrm{~A}, \mathrm{~m}-\mathrm{q}$ ).

Some of the phenotypic features have been previously associated to the haploinsufficiency of the nuclear factor I/A gene (NFIA, MIM 600727), mainly corpus callosum hypoplasia/absence, hydrocephalus/ventriculomegaly, and urinary tract malformations, since the Nfia knockout mice showed agenesis of corpus callosum, communicating hydrocephalus, female subfertility, and male sterility [das Neves et al., 1999; Lu et al., 2007]. But, more recently, a single patient with craniosynostosis and a family with phenotypic 1p32.p31 syndrome and craniosynostosis were found to have microdeletions which affected only NFIA, thus suggesting that this gene was also important in craniofacial development [Rao et al., 2014; Nyboe et al., 2015].

Here, we report the identification of an $\sim 18.6-\mathrm{Mb}$ deletion of chromosome 1p31.1p31.3 involving the loss of 68 genes in a patient referred with craniosynostosis and multiple congenital malformations. We subsequently review the available literature regarding chromosome 1p31.1p31.3 deletions.

\section{Clinical Report}

A 27-day-old infant born to nonconsanguineous parents, who have an older healthy son, was referred for genetic testing. The mother was 19 years old at the time of birth. Prenatal ultrasound revealed polyhydramnios, intrauterine growth restriction, hydrocephalus with enlargement of lateral ventricles, a prominent midfrontal line, exophthalmos, hypotelorism, and micrognathia. Amniocentesis was performed, and a normal female karyotype was obtained. The family refused further testing.

An uneventful caesarean section was performed at 38 weeks of gestation. Birth weight 2,590 g (P3-P10), length $45 \mathrm{~cm}$ (P10-P25), and head circumference $35.5 \mathrm{~cm}$ (P75-P90). Physical examination revealed a cloverleaf skull, hypotelorism and severe exophthalmos with absence of eyelids, ectopia lentis, sclerocornea, cleft palate, low-set ears, and cutis laxa. The hemogram, chemical analysis, and arterial blood gases were normal. Radiographic analysis showed craniosynostosis. Brain echography revealed biventricular enlargement with collapse of the third and fourth ventricles, small posterior fossa, and focal intracerebral hemorrhage in the left temporal lobe. Computed axial brain tomography showed pansynostosis with a cloverleaf skull, including sutures at the base of the cranium; hypotelorism, and obstructive hydrocephalus with enlargement of the temporal regions (fig. 2). MRI of the brain revealed severe enlargement of temporal ventricles with 2 hemorrhagic cortical foci in the right temporal and frontal regions, and
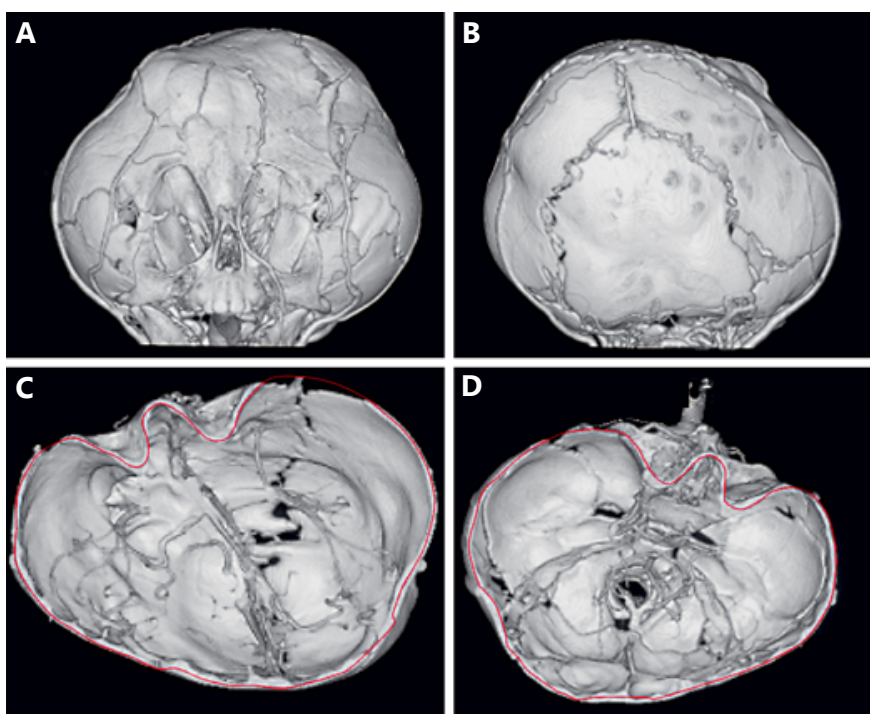

Fig. 2. Computed axial tomography image of the proband at 28 days, anterior (A) and posterior projection (B) and axial section (C, D) showing cranial asymmetry with a cloverleaf-shaped skull.

subcortical subacute hemorrhage. Echocardiogram was normal. Renal ultrasound assessment showed bilateral hypoplasia with abnormal cortical echogenicity and altered corticomedullary differentiation.

The patient was referred for array analysis and screening of craniosynostosis-related genes. The girl died at 7 months of age after a respiratory tract infection.

\section{Materials and Methods}

DNA from the patient was screened for mutations in the craniosynostosis genes as previously described [Paumard-Hernández et al., 2015] and for chromosomal rearrangements using the Infinium Cyto-SNP-850K BeadChip (Illumina), according to the manufacturer's instructions and analyzed on an Infinium iScan ${ }^{\mathrm{TM}}$ System platform (Illumina). The data was analyzed using the GenomeStudio ${ }^{\mathrm{TM}}$ software (Illumina). The deletion was confirmed by a chromosome 1 p31 custom-designed MLPA, including 11 probes spanning the region and 3 control probes (online suppl. table 1 ; for all suppl. material, see www.karger.com/doi/10.1159/000452609). The data was analyzed using the Coffalyser MLPA analysis program (MRC-Holland).

\section{Results}

The patient was referred for genetic testing of craniosynostosis-related genes. No mutations or deletions were detected in FGFR1, FGFR2, FGFR3, TWIST, EFNB1, and 
TCF12 [Paumard-Hernández et al., 2015]. Subsequently, a SNP-array was performed.

A deletion of chromosome 1p31.1p31.3 was detected using the CytoSNP-850K array, hg19:1(63,871,758$82,484,133) \times 1$ (fig. 1B). This $~ 18.6-\mathrm{Mb}$ deletion included 68 OMIM protein coding genes (http://www.ncbi.nlm. nih.gov/omim; online suppl. table 2). NFIA was not included in the deletion interval. No other copy number variation (CNV) was detected with the array. A customdesigned MLPA confirmed the presence of the deletion (fig. 1C) and verified that 2 copies of NFIA were present. Both healthy parents refused genetic testing.

\section{Discussion}

We have identified an $\sim 18.6-\mathrm{Mb}$ deletion of $1 \mathrm{p} 31.1 \mathrm{p} 31.3$ in a newborn patient with multiple and severe congenital malformations, including craniosynostosis, cutis laxa, dysmorphic facial features, and abnormalities of the CNS, eye, and kidneys. A total of 68 OMIMlisted genes were located within the deleted region.

To date, 17 cases with deletions of varying size of $1 \mathrm{p} 31.1 \mathrm{p} 31.3$ have been reported in the literature or in the DECIPHER database (https://decipher.sanger.ac.uk/). Clinical features included developmental delay, seizures, CNS malformations, macrocephaly ( $40 \%$ of the cases), elongated or rounded facies with a prominent nose, micro/retrognathia, half-opened mouth, short neck, congenital heart malformations, hernia, hand/foot malformations, renal anomalies, abnormal external genitalia, joint hyperlaxity, and cutis laxa (online suppl. table 3 ). Two were intragenic deletions of NFIA, identified in individuals with craniosynostosis, and it was hypothesized that this clinical feature was due to NFIA haploinsufficiency [Rao et al., 2014; Nyboe et al., 2015]. A 1p34.1p31 inversion-duplication was also described in a patient with craniosynostosis, but the breakpoints were not characterized [Garcia-Heras et al., 1999]. However, the patient reported in this study has a normal copy number for this gene; thus, it is unlikely that this gene is the cause for the craniosynostosis observed in our patient.

It has been reported that $7.5 \%$ of the cases with craniosynostosis involving one suture display a previously unreported CNV [Mefford et al., 2010]. In our laboratory, $3.5 \%$ of the cases with one or more affected sutures have a pathogenic CNV (unpublished data). We then tried to determine which gene may be responsible for the craniosynostosis. We searched the DECIPHER database for craniosynostosis cases and deletions in chromosome 1 and found an individual with a smaller $1 \mathrm{p} 31.3$ deletion including 10 OMIM genes (IL23R, IL12RB2, SERBP1, DDIT1, GNG12, GNG12AS1, DIRAS3, WLS, RPE65, and $D E P D C 1$ ) in a father and his son (DECIPHER 277832) both with sagital craniosynostosis, hypercalcemia, and postnatal growth delay. Some of these genes have been reported as partially or completely deleted (in heterozygous state) in the 'healthy control' population, with variable frequencies (MAF 0-0.03\%; online suppl. table 4). Only one of these genes, IL23R, encoding a subunit of the receptor for IL23A/IL23, could have a potential relationship with craniosynostosis. This protein pairs with IL12RB1 to conform the specific region of the heterodimeric interleukin (IL)-23 receptor required for signaling via the JAK/STAT pathway. IL23R associates constitutively with Janus kinase 2 (JAK2) and can also bind to transcription activators STAT3, STAT1, STAT4, and STAT5 in a ligand-dependent manner [Parham et al., 2002]. Heterozygous STAT3 mutations are associated with autosomal dominant hyper IgE syndrome (ADHIES), a primary immune deficiency. Craniosynostosis has been described in 4 ADHIES cases [Smithwick et al., 1978; Hoger et al., 1985]. Homozygous mutations in the interleukin 11 receptor alpha gene (IL11RA) were also identified in a family with Crouzon-like craniosynostosis [Keupp et al., 2013]. Thus, we propose that the craniosynostosis in our patient may be due to the heterozygous deletion of IL23R.

NFIA haploinsuficiency has also been proposed to be responsible for CNS malformations such as ventriculomegaly, hidrocephalus, corpus callosum anomalies, tethered spinal cord, or Chiari malformation which were observed in 5 cases with deletions overlapping the region deleted in our patient [Lu et al., 2007]. Indeed, the Nfia knockout mouse $\left(\mathrm{Nfia}^{-/}\right)$shows a lethality rate of $>95 \%$, with hydrocephalus and corpus callosum agenesis, whilst the heterozygous mice $\left(\mathrm{Nfia}^{+/-}\right)$display urinary tract and CNS malformations [das Neves et al., 1999; Lu et al., 2007]. However, despite our patient shows ventriculomegaly and hydrocephalus, the deletion does not include NFIA. The breakpoint is $>1 \mathrm{Mb}$ from this gene; thus, the disruption of regulatory elements is also unlikely. But since we are unable to analyze NFIA expression in this patient, our opinion is that another gene within the deleted interval may be the cause of the CNS malformations.

Of the 68 affected genes, 12 of them have a MIM MORBID ID: FOXD2, ALG6, PGM1, DNAJC6, LEPR, SLC35D1, IL23R, RPE65, CTH, TNNI3K, ACADM, and NEXN. We performed an evaluation of the genes deleted in our patient using the haploinsufficiency score [Huang et al., $2010]$. Of the 68 OMIM protein coding genes, 10 were 
found to have a high haploinsufficiency score: $P D E 4 B$, GADD45, LRRRC7, ZRANB2, NEGR1, LHX8, ST6GALNAC3, ZZZ3, FUBP1, and ELTD1. The frequencies of reported CNVs for these genes are variable and are present in $0-0.03 \%$ of the control population, according to Exome Aggregation Consortium (http://exac.broadinstitute.org/; online suppl. table 5).

FOXD2 is the kidney-expressed human forkhead gene; thus, it could be a candidate for the kidney malformations presented by our patient because this gene is transcribed exclusively in the kidney [Ernstsson et al., 1997].

The presence of a cleft palate combined with microcephaly and severe learning difficulties has also been reported in a patient with a smaller chromosome 1 deletion [Shaw-Smith et al., 2004]. LHX8 haploinsufficiency was present in both cases. This gene encodes a transcriptional regulator of the family LIM-homeobox, which are expressed in the first branchial arch and the basal forebrain [Grigoriou et al., 1998]. Mice mutant for $L h x 8$ revealed a crucial role for LHX8 in palatogenesis: the bilateral primordial palatal shelves are formed and elevated correctly, but they often failed to make contact and fuse properly, resulting in a cleft secondary palate [Zhao et al., 1999]. Although this gene is highly expressed in skin and connective tissue, there is no clear relation between its haploinsufficiency and the cutis laxa phenotype described in our patient. The gene that may be responsible for this clinical feature is ST6GALNAC3, which has been intra- genically partially deleted in 1 patient who presented excessively wrinkled skin amongst other anomalies (DECIPHER 249129). The remaining dose-responsive genes have been linked to the clinical features observed in patients with chromosome 1p32p21 deletion syndrome.

In summary, we report a chromosome $1 \mathrm{p} 31.1 \mathrm{p} 31.3$ deletion encompassing $\sim 18,6 \mathrm{Mb}$ and including 68 OMIM genes in a patient with multiple malformations. Using clinical data from other chromosome 1 deletion patients and mice models, we propose that IL23R may be responsible for the craniosynostosis, whilst various deleted genes may explain the other clinical features.

\section{Acknowledgment}

This work was supported in part by the Spanish Ministry of Research, Development and Innovation (MICINN: SAF201230871, SAF2015-66831-R).

\section{Statement of Ethics}

The parents have given written informed consent, and ethical approval was obtained from the local ethics committee.

\section{Disclosure Statement}

The authors declare no conflicts of interest.

\section{References}

Bene M, Duca-Marinescu A, Ioan D, Maximilian C: De novo interstitial deletion del(1) (p21p32). J Med Genet 16:323-327 (1979).

Campbell CGN, Wang H, Hunter GW: Interstitial microdeletion of chromosome $1 \mathrm{p}$ in two siblings. Am J Med Genet 111:289-294 (2002).

Chen CP, Su YN, Chen YY, Chern SR, Liu YP, et al: Chromosome 1p32-p31 deletion syndrome: prenatal diagnosis by array comparative genomic hybridization using uncultured amniocytes and association with NFIA haploinsufficiency, ventriculomegaly, corpus callosum hypogenesis, abnormal external genitalia, and intrauterine growth restriction. Taiwan J Obstet Gynecol 50:345-352 (2011).

das Neves L, Duchala CS, Tolentino-Silva F, Haxhiu MA, Colmenares C, et al: Disruption of the murine nuclear factor I-A gene (Nfia) results in perinatal lethality, hydrocephalus, and agenesis of the corpus callosum. Proc Natl Acad Sci USA 96:11946-11951 (1999).
Ernstsson S, Betz R, Lagercrantz S, Larsson C, Ericksson S, et al: Cloning and characterization of freac-9 (FKHL17), a novel kidneyexpressed human forkhead gene that maps to chromosome 1p32-p34. Genomics 46:78-85 (1997).

Garcia-Heras J, Corley N, Garcia MF, Kukolich MK, Smith KG, Day DW: De novo partial duplications 1p: report of two new cases and review. Am J Med Genet 82:261-264 (1999).

Gillberg C, FitzPatrick D: Case report: further evidence for a recognizable syndrome caused by deletion of 1p31. Adv Clin Neurosci Rehabil 3:16-18 (2010).

Grigoriou M, Tucker AS, Sharpe PT, Pachnis V: Expression and regulation of $L h x 6$ and $L h x 7$, a novel subfamily of LIM homeodomain encoding genes, suggests a role in mammalian head development. Development 125:20632074 (1998).

Hoger PH, Boltshauser E, Hitzig WH: Craniosynostosis in hyper-IgE syndrome. Eur J Pediatr 144:414-417 (1985).
Huang N, Lee I, Marcotte EM, Hurles ME: Characterising and predicting haploinsufficiency in the human genome. PLoS Genet 6:e1001154 (2010)

Ji J, Salamon N, Quintero-Rivera F: Microdeletion of 1p32-p31 involving NFIA in a patient with hypoplastic corpus callosum, ventriculomegaly, seizures and urinary tract defects. Eur J Med Genet 57:267-268 (2014).

Keupp K, Li Y, Vargel I, Hoischen A, Richardson $\mathrm{R}$, et al: Mutations in the interleukin receptor IL11RA cause autosomal recessive Crouzonlike craniosynostosis. Mol Genet Genomic Med 1:223-237 (2013).

Labonne JD, Shen Y, Kong IK, Diamond MP, Layman LC, Kim HG: Comparative deletion mapping at 1p31.3-p32.2 implies NFIA responsible for intellectual disability coupled with macrocephaly and the presence of several other genes for syndromic intellectual disability. Mol Cytogenet 9:24 (2016). 
Lai MM, Robards MF, Berry AC, Fear CN, Hart C: Two cases of interstitial deletion 1p. J Med Genet 28:128-130 (1991).

Lu W, Quintero-Rivera F, Fan Y, Alkuraya FS, Donovan DJ, et al: NFIA haploinsufficiency is associated with a CNS malformation syndrome and urinary tract defects. PLoS Genet 3:e80 (2007).

Mefford HC, Shafer N, Antonacci F, Tsai JM, Park SS, et al: Copy number variation analysis in single-suture craniosynostosis: multiple rare variants including RUNX2 duplication in two cousins with metopic craniosynostosis. Am J Med Genet A 152A:2203-2210 (2010).

Mircher C, Rethore MO, Lespinasse J, Fert-Ferrer S, Lundsteen C, Kirchoff M: Interstitial deletion of the short arm of chromosome 1: attempt to establish a clinical phenotype (46,XX,del (1)(p22p32)). Am J Med Genet A 118A:176-179 (2003).

Nyboe D, Kreiborg S, Kirchhoff M, Hove HB: Familial craniosynostosis associated with a microdeletion involving the NFIA gene. Clin Dysmorphol 24:109-112 (2015).
Parham C, Chirica M, Timans J, Vaisberg E, Travis $\mathrm{M}$, et al: A receptor for the heterodimeric cytokine IL-23 is composed of IL-12R-beta-1 and a novel cytokine receptor subunit, IL23R. J Immunol 168:5699-5708 (2002).

Paumard-Hernández B, Berges-Soria J, Barroso E, Rivera-Pedroza CI, Pérez-Carrizosa V, et al: Expanding the mutation spectrum in 182 Spanish probands with craniosynostosis: identification and characterization of novel TCF12 variants. Eur J Hum Genet 23:907-914 (2015).

Petersen MB, Warburg M: Interstitial deletion 1p in a 30 year old woman. J Med Genet 24:229231 (1987).

Rao A, O’Donnell S, Bain N, Meldrum C, Shorter $\mathrm{D}$, Goel H: An intragenic deletion of the NFIA gene in a patient with a hypoplastic corpus callosum, craniofacial abnormalities and urinary tract defects. Eur J Med Genet 57:65-70 (2014).

Shaw-Smith C, Redon R, Rickman L, Rio M, Willatt L, et al: Microarray based comparative genomic hybridization (array-CGH) detects submicroscopic chromosomal deletions and duplications in patients with learning disability/mental retardation and dysmorphic features. J Med Genet 41:241-248 (2004).
Sivasankaran S, Ho NK, Knight L: De novo interstitial deletion of chromosome $1 \mathrm{p}$ with absent corpus callosum - a case report. Ann Acad Med Singapore 26:507-509 (1997).

Smithwick EM, Finelt M, Pahwa S, Good RA, Naspitz CK, et al: Cranial synostosis in Job's syndrome. Lancet 1:826 (1978).

Tassano E, Gamucci A, Celle ME, Ronchetto P, Cuoco C, Gimelli G: Clinical and molecular cytogenetic characterization of a de novo interstitial $1 \mathrm{p} 31.1 \mathrm{p} 31.3$ deletion in a boy with moderate intellectual disability and severe language impairment. Cytogenet Genome Res 146:39-43 (2015).

Zhao Y, Guo YJ, Tomac AC, Taylor NR, Grinberg $A$, et al: Isolated cleft palate in mice with a targeted mutation of the LIM homeobox gene lhx8. Proc Natl Acad Sci USA 96:1500215006 (1999).

Zinner SH, Batanian JR: Second reported patient with $\operatorname{del}(1)(\mathrm{p} 32.1 \mathrm{p} 32.3)$ and similar clinical features suggesting a recognizable chromosomal syndrome. Am J Med Genet A 122A:164-167 (2003). 